\title{
MARCH6 promotes hepatocellular carcinoma development through up- regulation of ATF2
}

Jie Sun ${ }^{1 \dagger}$, Zheng Dong ${ }^{1 \dagger}$, Zhengyao Chang $^{2+}$, Hongfei Liu ${ }^{3}$, Qiyu Jiang ${ }^{4}$, Deyuan Zhang ${ }^{1}$, Shanshan Lu', Xiaodong Jia ${ }^{1}$, Dawei $\mathrm{Wu}^{1}$, Aaron $\mathrm{Ge}^{1}$, Pan Zhao ${ }^{5^{*}}$, Jing Wang ${ }^{6^{*}}$ (D) and Yinying Lu ${ }^{1^{*}}$

\begin{abstract}
Background: Hepatocellular carcinoma (HCC) is a common cause of cancer mortality worldwide. Recent studies have shown that the polytopic enzyme membrane associated ring-CH-type finger 6 (MARCH6) participates in tumorigenesis, but its function in HCC development needs to be investigated. This study aimed to explore the role of MARCH6 in HCC.

Methods: Expression of MARCH6 in human HCC samples was checked by immunohistochemical staining assay. Clinical relevance of MARCH6 and activating transcription factor 2 (ATF2) was analyzed from TCGA database. CCK-8, EdU staining, colony formation and transwell were performed to assess cell proliferation, growth and migration. Xenografted tumorigenesis was used to examine in vivo role MARCH6. Immunoblotting was applied to detect protein abundance.

Results: We found that MARCH6 expression was elevated in human HCC samples. Over-expression of MARCH6 was associated with poor prognosis of HCC patients. Up-expression of MARCH6 promoted cell growth and migration of HCC cells. In contrast, the HCC cell growth and migration were suppressed by MARCH6 knockdown. Furthermore, the DNA synthesis was enhanced by MARCH6. The expression of ATF2 was potentiated by MARCH6 overexpression, while it was suppressed by MARCH6 silencing. TCGA database showed positive correlation between the expression of MARCH6 and ATF2. Importantly, ATF2 expression contributed to the oncogenic function of HCC cells.

Conclusion: Our findings suggest that MARCH6-mediated ATF2 up-regulation contributes to HCC development. MARCH6 may be a promising target for the diagnosis and treatment of HCC.
\end{abstract}

Keywords: Hepatocellular carcinoma, MARCH6, Proliferation, Migration, ATF2

\footnotetext{
* Correspondence: doczhaopan@126.com; JingWang@bmi.ac.cn;

Luyinying1973@163.com

${ }^{\dagger}$ Jie Sun, Zheng Dong and Zhengyao Chang contributed equally to this work.

${ }^{5}$ Department of Infectious Diseases, The Fifth Medical Center of PLA General Hospital, Beijing 100039, China

${ }^{6}$ State key Laboratory of Toxicology and Medical Countermeasures, Beijing Institute of Pharmacology and Toxicology, Beijing 100850, China

${ }^{1}$ Comprehensive Liver Cancer Center, The Fifth Medical Center of PLA General Hospital, Beijing 100039, China

Full list of author information is available at the end of the article
}

(C) The Author(s). 2021 Open Access This article is licensed under a Creative Commons Attribution 4.0 International License, which permits use, sharing, adaptation, distribution and reproduction in any medium or format, as long as you give appropriate credit to the original author(s) and the source, provide a link to the Creative Commons licence, and indicate if changes were made. The images or other third party material in this article are included in the article's Creative Commons licence, unless indicated otherwise in a credit line to the material. If material is not included in the article's Creative Commons licence and your intended use is not permitted by statutory regulation or exceeds the permitted use, you will need to obtain permission directly from the copyright holder. To view a copy of this licence, visit http://creativecommons.org/licenses/by/4.0/ The Creative Commons Public Domain Dedication waiver (http://creativecommons.org/publicdomain/zero/1.0/) applies to the data made available in this article, unless otherwise stated in a credit line to the data. 


\section{Background}

Hepatocellular carcinoma (HCC) is digestive malignancy with poor clinical outcomes and accounts for the second leading cause of cancer death worldwide [1,2]. Over the past decade, major advances have been obtained to understand the epidemiologic risk factors as well as the molecular mechanisms that drive HCC tumorigenesis [1, 2]. As ubiquitination of proteins mediated proteolytic degradation in post-translational level, increasing amount of evidence indicates that protein ubiquitination is involved in a multitude of processes, including endocytosis, DNA repair, signal transduction and tumorigenesis [3-6].

Protein ubiquitination is an important post-transcriptional modification that regulates protein stability. With the aid of E3 ligases, ubiquitin is ligated to its specific substrate [7]. As a subfamily of the RING-type E3 ubiquitin ligase, membrane associated ring- $\mathrm{CH}$-type finger $(\mathrm{MARCH})$ proteins are discovered as the human homologs of the viral membrane ubiquitin ligases K3 and K5 from Kaposi's sarcoma-associated herpesvirus [8]. Most MARCH family members have been indicated to be implicated in the development of human diseases $[9,10]$. Among them, MARCH6 was first identified as a TEB4, which mediates the basal and cholesterol-induced degradation of squalene epoxidase [11]. The stability of MARCH6 was regulated by ubiquitin-specific protease 19 [12]. Recent studies indicated that MARCH6 was positively corrected with androgen receptor (AR) gene expression, which shared common regulation with MARCH6 by the transcription factor $\mathrm{Sp} 1$ in prostate cancer [13]. Additional studies suggested that MARCH6 and IDOL E3 ubiquitin ligase circuit played a multifaceted role in the regulation of cholesterol homeostasis in hepatocytes [14]. However, the specific functions of MARCH6 in HCC remain unclear.

Activating transcription factor 2 (ATF2) is a transcription factors which belongs to activator protein 1 (AP-1) family [15]. Aberrant expression or activation of ATF2 regulates the expression of a wide range of genes, which are involved in cell survival, proliferation, growth, apoptosis and DNA damage response, thereby playing an important role in cancer progression [16, 17]. For example, ATF2 functions as an oncogene in kinds of cancer types, including breast cancer [18], melanoma [19], lung cancer [20] and colorectal cancer [21]. Although it is well known that ATF2 contributes to carcinogenesis through regulating the expression of downstream targets, the upstream modulator of ATF2 and its significance in HCC remain to be determined.

Herein, we designed the present study to explore the role of MARCH6 in HCC. To this aim, we constructed MARCH6 knockdown and overexpressing HCC cells and examined $\mathrm{HCC}$ cell proliferation, migration and tumorigenesis. Our study not only identified MARCH6 as an oncogene, but also demonstrated ATF2 as an contributor of MARCH6 in HCC development.

\section{Methods \\ MARCH6 expression analysis from TCGA database}

MARCH6 transcripts in human HCC samples (tumor, $n=373$; normal, $n=50$ ) were analyzed from The Cancer Genome Atlas (TCGA). For overall and disease free survival analysis, $\mathrm{HCC}$ patients were subdivided into MARC H6 high expression group and low expression group. Group cutoff was set by quartile. The study was approved by the ethics committees of the Fifth Medical Center of Chinese PLA General Hospital.

\section{Immunohistochemical staining of MARCH6}

10 pairs of $\mathrm{HCC}$ and adjacent normal tissues were used in this study. 5 um of paraffin-embedded tissues were subjected to immunohistochemical staining of MARC H6, following the indicated protocols as described previously [22, 23]. Quantification of MARCH6 protein abundance was determined by image J software.

\section{Cell culture}

Huh7, HCCLM3 and MHCC97H cells were from Shanghai Zhong Qiao Xin Zhou Biotechnology Co.,Ltd. [24, 25]. Cells were cultured in DMEM (Invitrogen) in a $37{ }^{\circ} \mathrm{C}$ incubator with $5 \% \mathrm{CO}_{2}$, supplied with $10 \%$ fetal bovine serum (Gibco) and 1\% antibiotics (Corning).

\section{Lentivirus-mediated MARCH6 knockdown}

Lentivirus vector pLL3.7 was used for MARCH6 knockdown in $\mathrm{MHCC} 97 \mathrm{H}$ cells. The shRNA sequences were listed: shCtrl, 5' -TTCTCCGAACGTGTCACGT-3'; shMARCH6\#1, 5'-GCACACTGTGTGCATTCATCA-3'; shMARCH6\#2, 5'-GCTTGTGGTCTCTATGTTTGC-3'. shATF2, 5' -GGAGCCTTCTGTTGTAGAAAC-3'.

\section{MARCH6 over-expression}

The CDS sequence of MARCH6 and ATF2 was constructed into $\mathrm{pCDH}$ lentivirus vector. Packaging vectors and $\mathrm{pCDH}$ vectors were transfected into $293 \mathrm{~T}$ cells. The virus were collected $48 \mathrm{~h}$ later and used to infect HCC cells.

\section{Cell proliferation}

CCK-8 was applied to determine cell proliferation. Equal number of the indicated cells was seed into the 96-well plates, which contained $200 \mu \mathrm{l}$ culture medium. At indicated time, $20 \mu \mathrm{l} \mathrm{CCK}-8$ regent (Beyotime) was incubated with the culture medium at $37{ }^{\circ} \mathrm{C}$ for three hours. Cell viability was determined by measuring OD450 on a micro-plate reader.

\section{Colony formation}

For MHCC97H cells, a total 10,000 shCtrl, shMARCH6-1 and shMARCH6-1 + ATF2 MHCC97H cells were plated. For Huh7 and HCCLM3 cells, a total 500 Ctrl, MARCH6 
and MARCH6 + shATF2 Huh7 and HCCLM3 cells were plated. Colonies were grown for 10 days and then were stained by crystal violet $(0.1 \%)$. The images were photographed by the camera.

\section{EdU staining}

BeyoClick $^{\mathrm{ma}}$ EdU Cell Proliferation Kit was applied to detect EdU cooperation. In brief, equal number of $\mathrm{MHCC} 97 \mathrm{H}$ and Huh7 cells was seeded onto the coverslip in the 6-well plates. $16 \mathrm{~h}$ later, the cells were stained with EdU and DAPI, following the protocols.

\section{Transwell}

Cell migration was determined by using transwell assay, following the protocols. For MHCC97H cells, a total of $10^{6}$ shCtrl, shMARCH6-1 and shMARCH6-1 + ATF2 MHCC97H cells were seeded per well. For Huh7 and HCCLM3 cells, a total of $5 \times 10^{5} \mathrm{Ctrl}$, MARCH6 and MARCH6 + shATF2 Huh7 and HCCLM3 cells were seeded per well. $24 \mathrm{~h}$ later, cell migration was detected by staining with $0.1 \%$ crystal violet.

\section{Immunoblotting}

Proteins were extracted using lysis buffer. Protein concentration was assessed by BCA method (Beyotime). Total proteins was subjected to sodium dodecyl sulfatepolyacrylamide gel electrophoresis (SDS-PAGE) and transferred to PVDF membranes (Millipore, USA), then blocked with $5 \%$ skim milk. After blocking, the membranes were incubated with primary antibodies followed by incubation with secondary antibodies. The signal was detected by chemiluminescence (Thermo Fisher Scientific). Primary antibodies against MARCH6 (ab183533) and ATF2 (ab47476) were purchased from Abcam. GAPD H (60004-1-Ig) antibody was purchased from Proteintech.

\footnotetext{
Animal model

A total of $1 \times 10^{6}$ MHCC97H cells expressing shCtrl or shMARCH6 lentivirus were subcutaneously implanted into BALB/c nude mice (female, 4-5-weeks old, randomly grouped for two groups, $n=5,16-18 \mathrm{~g}$ per mice), which were purchased from Charles River (Beijing, China). The mice were kept under SFP room with 12-h light/12-h dark cycles. Tumor volume was monitored twice a week. The mice were anesthetized by pentobarbital sodium and were euthanized by carbon dioxide 37 days after implantation. Animal experiments were performed according the provisions of the Declaration of Helsinki and were approved by the Ethical Committee for Animal Experimentation of The Fifth Medical Center of PLA General Hospital.
}

\section{Statistical analysis}

Statistical analysis was performed by SPSS version 16.0 software. Data were shown as the mean \pm SEM of three independent experiments. Group comparisons were performed using $t$ test or one-way ANOVA. Spearman correlation analysis was performed to study the correlation between MARCH6 and oncogenes in cancer growth and metastasis. Kaplan-Meier analysis of event or event-free rates was performed and statistical differences were evaluated by the log-rank test. Tests were two-sided and a probability $(P)$ value of less than 0.05 was considered statistically significant.

\section{Results}

\section{MARCH6 is highly expressed in human HCC cells}

Firstly, we evaluated the clinical relevance of MARCH6 from TCGA database. We found that MARHC6 abundance was elevated in HCC samples (Fig. 1A). Furthermore, the overall survival and disease-free survival were both shorter in HCC patients who had MARCH6 high level compared to those who had MARCH6 low level $(P<0.05$, Fig. 1B). Nevertheless, MARCH6 expression was not significantly associated with HCC patients' $T$ stage and grade (Table 1). Then, we performed immunohistochemical staining of MARCH6. As shown in Fig. 1C, MARCH6 was up-regulated in HCC tissues compared to adjacent normal samples. Furthermore, Spearman correlation analysis in TCGA HCC tissues revealed that MARCH6 was positively correlated with MKI67, CTNNB1, CDH2, FN1 and WNTSA, which were demonstrated as oncogene in cancer growth and metastasis (Supplementary Fig. 1). By contrast, MARCH6 was negatively associated with BAD and BAX, which played an important role in apoptosis (Supplementary Fig. 1). Taken together, MARCH6 was a potential oncogene for HCC.

\section{MARCH6 increases the proliferation, growth and DNA synthesis of HCC cells}

To study the function of MARCH6, we firstly checked the expression of MARCH6 in three HCC cell lines, including Huh7, HCCLM3 and MHCC97H. The expression of MARCH6 was highest in MHCC97H cells as compared with Huh7 and HCCLM3 cells (Fig. 2A). We therefore infected MHCC97H cells with MARC H6 knockdown lentivirus and Huh7 or HCCLM3 cells with MARCH6 overexpressing lentivirus. Immunoblotting results showed that MARCH6 was efficiently knocked down in MHCC97H cells and was efficiently overexpressed in Huh7 and HCCLM3 cells (Fig. 2B). CCK assay demonstrated that MARCH6 silencing led to reduced cell viability of MHCC97H cells (Fig. 2C). By contrast, MARCH6 overexpression promoted the 
a

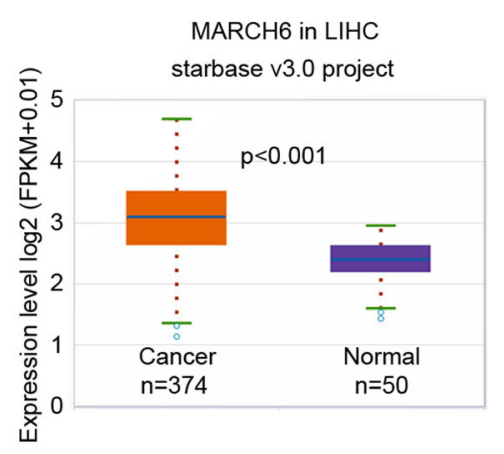

b

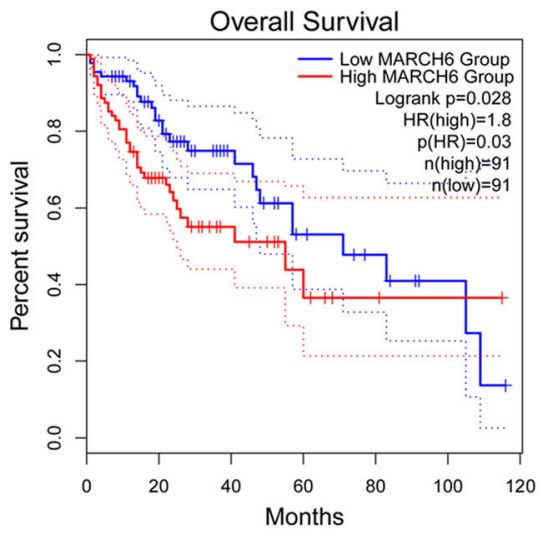

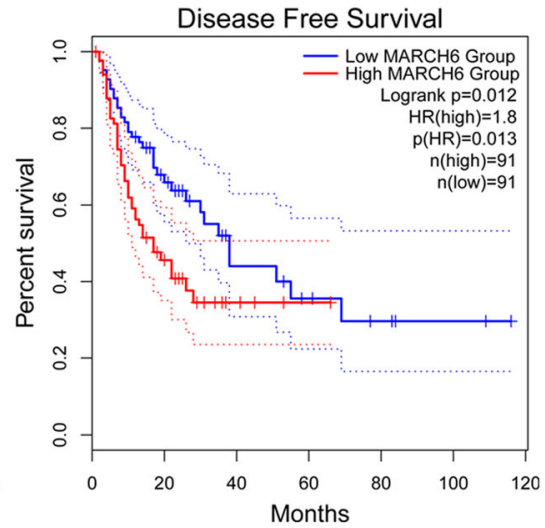

C
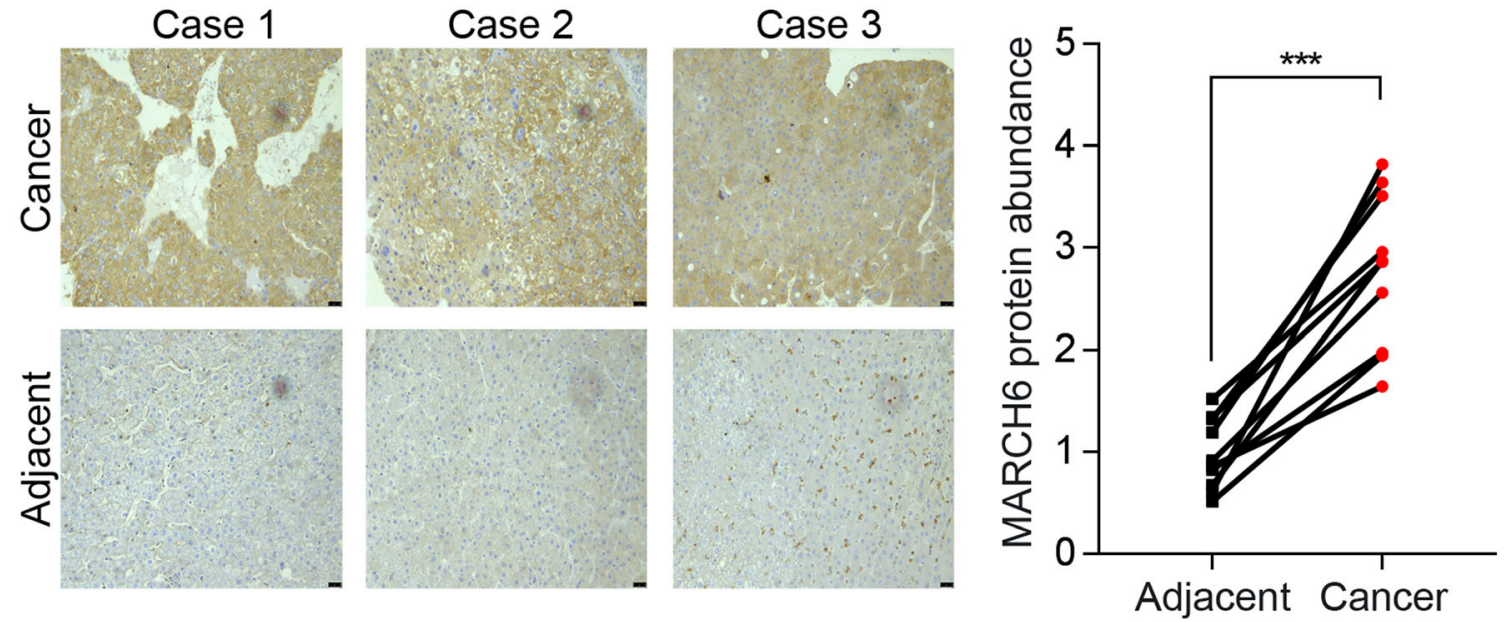

Fig. 1 MARCH6 is over-expressed in HCC tissues. (a) TCGA database showed that MARCH6 was up-regulated in HCC samples. $P<0.001$. (b) The overall and disease-free survival was determined in HCC patients who had MARCH6 high and low expression. Cutoff-High is $75 \%$ and Cutoff-Low is $25 \%$. $n=91$ for each group. $P<0.05$. (c) Immunohistochemical analysis of MARCH6 in HCC and adjacent tissues. Scale bar, 25 um. ${ }^{* *} P<0.001$

Table 1 Correlation between patients' characteristics and MARC H6 expression in LIHC from TCGA database

\begin{tabular}{llllll}
\hline Characteristics & & \multicolumn{2}{l}{ MARCH6 } & Total & P value \\
\cline { 3 - 4 } & & High & Low & & \\
\hline Sex & Male & 124 & 126 & 250 & .883 \\
& Female & 61 & 60 & 121 & \\
Total & & 185 & 186 & 371 & \\
Age & $\mathbf{5} 65$ & 124 & 108 & 232 & .085 \\
& $>65$ & 61 & 77 & 138 & \\
Total & & 185 & 185 & 370 & \\
T stage & T1/2 & 134 & 141 & 175 & .401 \\
& T3/4 & 50 & 43 & 93 & \\
Total & & 184 & 184 & 368 & \\
Grade & G1/2 & 108 & 124 & 232 & .083 \\
& G3/4 & 75 & 59 & 134 & \\
Total & & 183 & 183 & 366 & \\
\hline
\end{tabular}

proliferation of Huh7 and HCCLM3 cells (Fig. 2D). DNA synthesis is a biomarker for cell proliferation. We performed EdU staining in HCC cells and found that the number of EdU positive cells was reduced but increased in MARCH6 knockdown and overexpressed HCC cells (Fig. 2D and E), respectively. Collectively, MARCH6 has oncogenic function in HCC cells.

MARCH6 silencing retards the tumor growth of HCC cells To study the effect of MARCH6 silencing on tumorigenesis, shCtrl or shMARCH6 MHCC97H cells were subcutaneously transplanted into the right arms of nude mice ( $n=5$ per group). The results showed that MARC H6 knockdown in MHCC97H cells inhibited the tumorigenesis and growth (Fig. 3). Thus, MARCH6 is critical for HCC growth. 


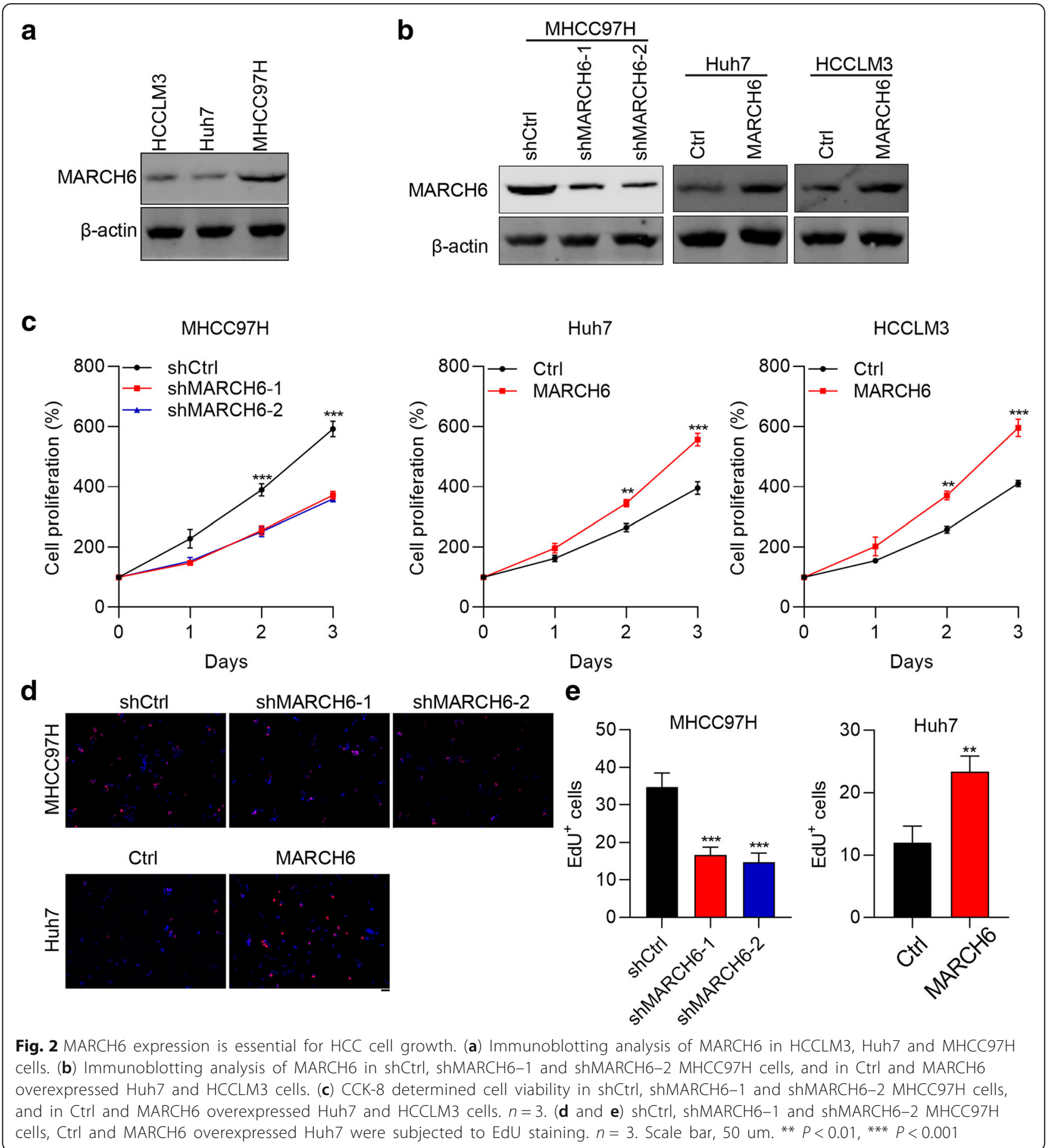

MARCH6 up-regulates ATF2 in HCC cells

To investigate the molecular mechanism underlying how MARCH6 promotes HCC, we analyzed the most correlated mRNAs from TCGA database. Among the database, we found that ATF2 was highly associated with MARCH6 expression (Fig. 4A). And the transcription level of ATF2 in HCC tumor sample was higher than the normal tissues (Fig. 4B). In addition, ATF2 expression level was inversely correlated with the survival of HCC patients (Fig. 4C). We then checked the expression of ATF2 in MARCH6 knockdown and over-expressed cells. Protein expression of ATF2 was reduced and elevated in MARCH6 silenced and over-expressed HCC 


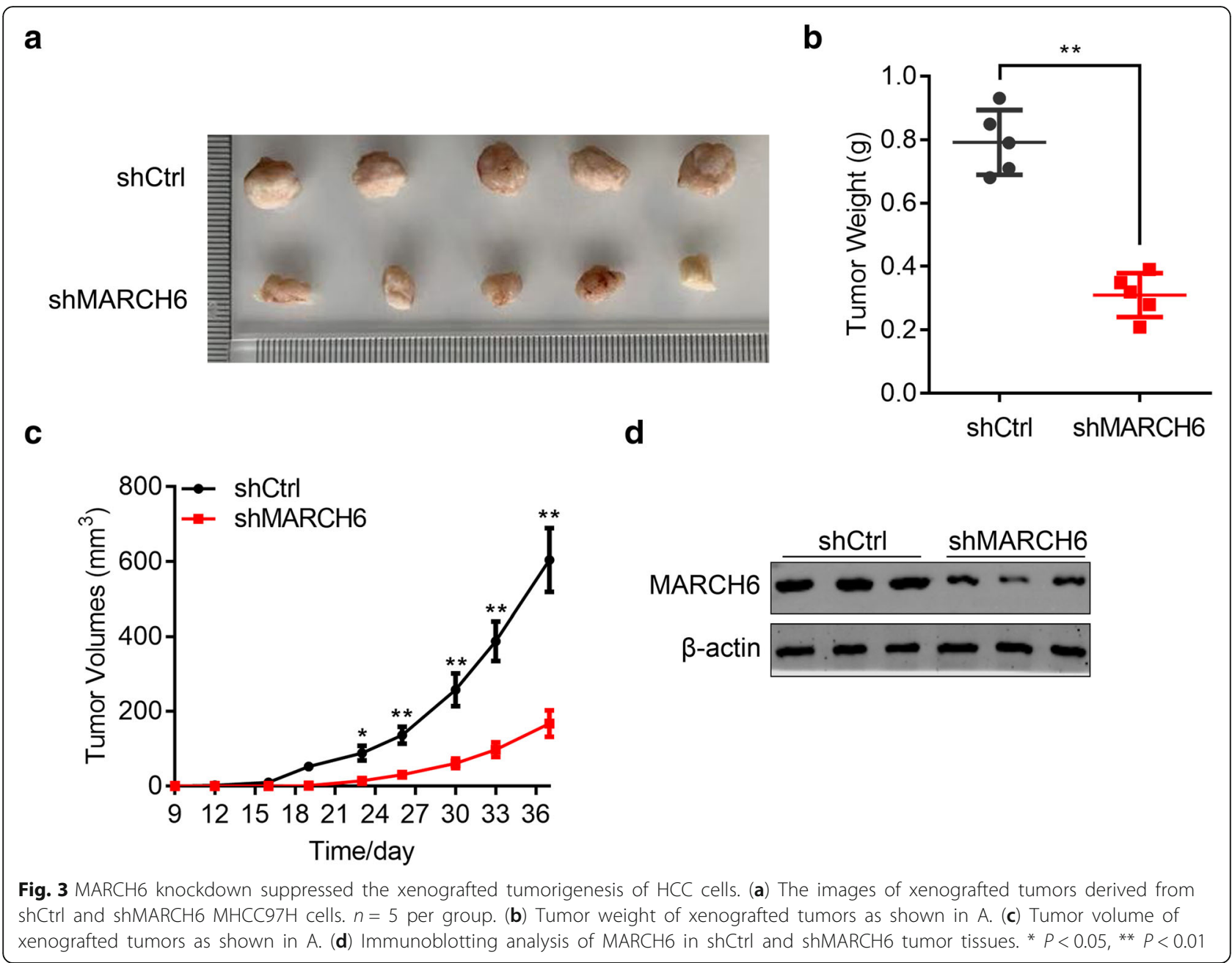

cells, respectively (Fig. 4D). These results suggest that MARCH6 could up-regulate ATF2 in HCC cells.

\section{Over-expression of ATF2 restores the growth and migration of $\mathrm{MHCC} 97 \mathrm{H}$ cells with down-regulation of MARCH6}

To explore whether MARCH6 knockdown suppresses the growth and migration of HCC cells through regulating the expression of ATF2, we infected MARCH6 silenced MHCC97H cells with ATF2 over-expressing lentivirus. As shown in Fig. 5A, ATF2 was efficiently over-expressed in MARCH6 silenced MHCC97H cells. CCK8 and colony formation results demonstrated that MARCH6 knockdown suppressed the proliferation and colony growth of $\mathrm{MHCC} 97 \mathrm{H}$ cells, which could be restored by ectopic expression of ATF2 (Fig. 5B and C). ATF2 over-expression also restored the migration capacity of $\mathrm{MHCC} 97 \mathrm{H}$ cells with silencing of MARCH6 (Fig. 5D). Our results indicate that MARCH6 up-regulation of ATF2 contributes to $\mathrm{HCC}$ cell growth and migration.

\section{Knockdown of ATF2 reverses the growth and migration of HCC cells with over-expression of MARC $\mathrm{H} 6$}

To further confirm the role of MARCH6/ATF2 axis in HCC cell growth and migration, we infected MARCH6 over-expressing Huh7 and HCCLM3 cells with ATF2 knockdown lentivirus. When MARCH6 over-expression promoted the expression of ATF2, knockdown lentivirus could significantly down-regulate ATF2 (Fig. 6A). ATF2 knockdown significantly reduced the proliferation and migration ability of Huh7 and HCCLM3 cells with overexpression of MARCH6 (Fig. 6B-E). Consistently, ATF2 down-regulation reversed the promoting role of MARC H6 up-regulation on the migration ability of Huh7 and HCCLM3 cells (Fig. 6D and E). Collectively, MARCH6/ 


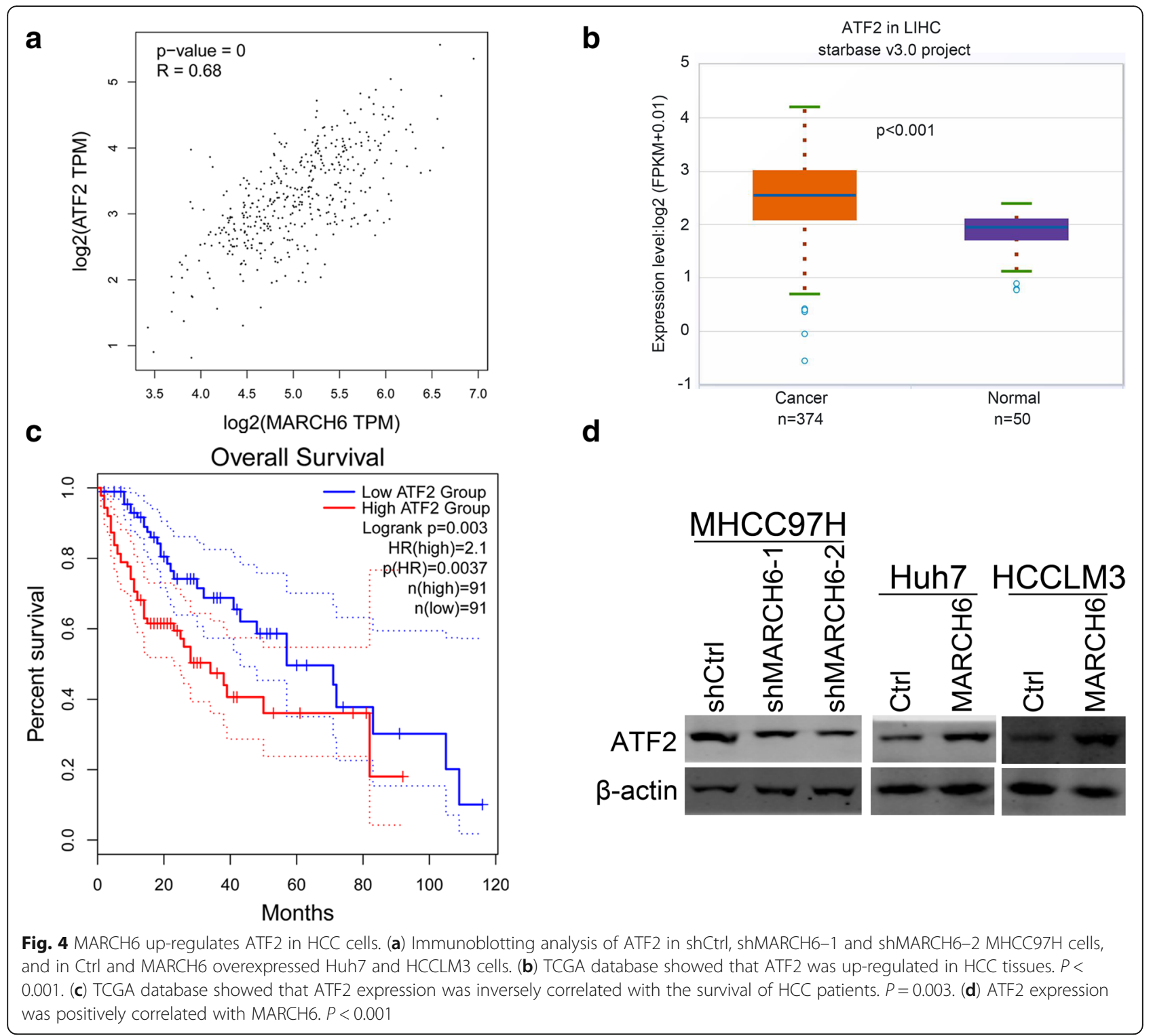

ATF2 axis is essential for HCC cell growth and migration.

\section{Discussion}

HCC is a common cause of cancer mortality [26]. Current data indicate that the emerging trends in HCC incidence is steadily increasing worldwide [27]. Till now, surgical resection, radiofrequency ablation and liver transplantation remain the widely used options for HCC patients, but with unsatisfactory clinical outcome due to a high risk of recurrence [28]. Studies have reported that the carcinogenesis and pathogenesis of $\mathrm{HCC}$ are involving in a wide variety of factors [29]. Notably, ubiquitination serves as a degradation mechanism of proteins, involved in a series of cellular processes, such as DNA damage repair, immune response and tumor metastasis, which is supported by multiple studies [30]. Among numbers of molecules and signal pathways attributed to protein ubiquitination, MARCH6 was suggested to function as a key player in modulating inflammatory response, lipoprotein uptake and tumor cell proliferation [14, 31, 32]. Despite that MARCH6 is an important E3 ligase, the specific functions of MARCH6 in HCC are poorly understood. Moreover, its mechanisms underlying the oncogene properties of MARCH6 in HCC were unexplored.

The expression level of MARCH6 is up-regulated in and knockdown of MARCH6 would inhibit the capacity of proliferation in breast cancer cells in previous study [31], but the expression pattern of MARCH6 in HCC remains unclear. Thus, we firstly examined MARCH6 expression and identified a strong correlation between MARCH6 expression and HCC patient's overall survival 


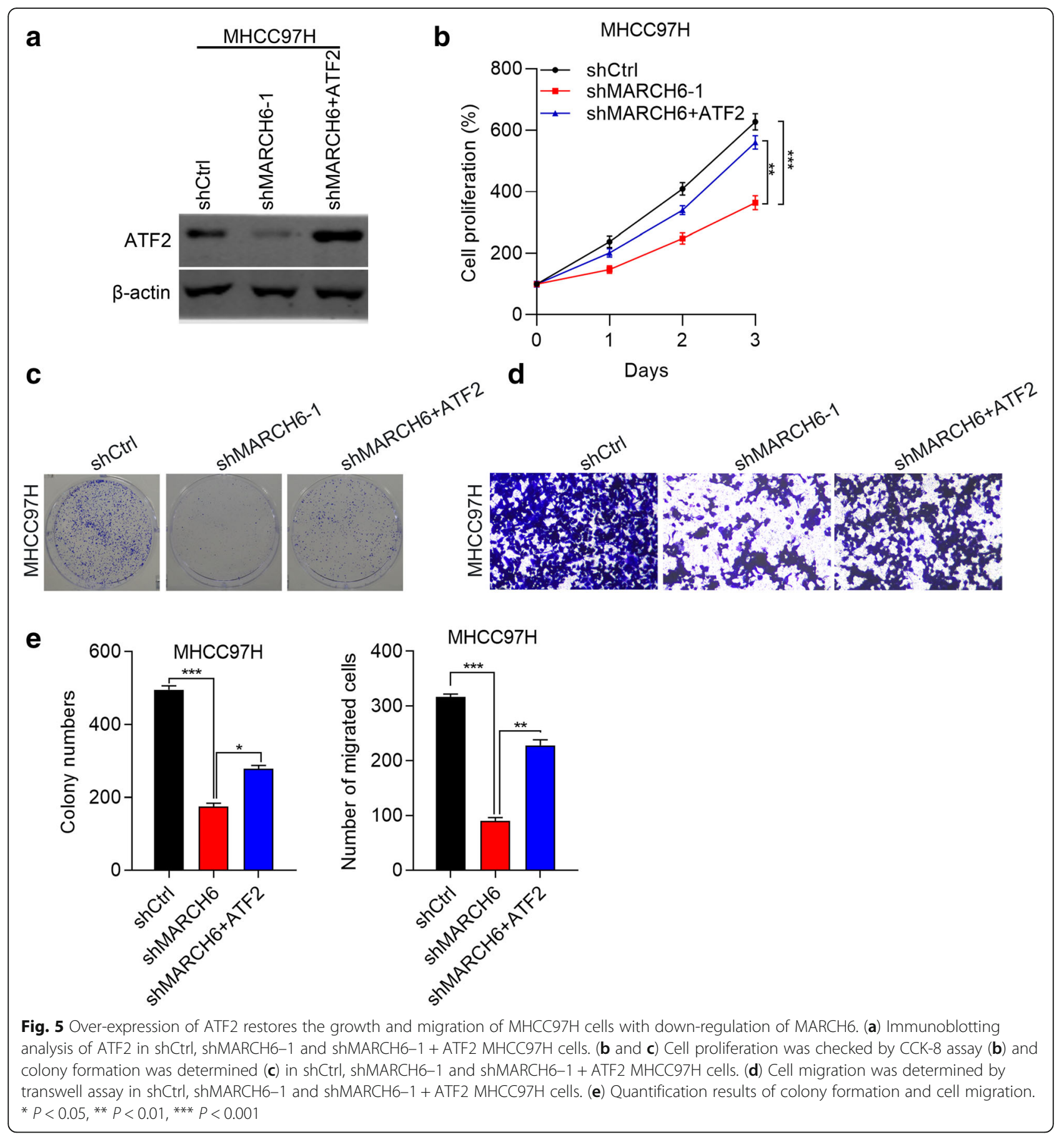

rate from clinical samples and public TCGA database. In addition, we presented evidence that MARCH6 knockdown attenuated the proliferative, colon formation capacity and DNA synthesis of HCC cell lines, as well as the ability of migration. Opposite results were obtained along with over-expression of MARCH6. Furthermore, to uncover the molecular mechanism responsible for the important function of MARCH6, we analyzed the most correlated mRNAs from TCGA database and found that
ATF2 is highly associated with the expression level of MARCH6, which suggested MARCH6 up-regulates ATF2 in HCC cells. Also we examined the significance of ATF2 and found ATF2 potentiates HCC cell function. Nevertheless, the detailed interaction between MARCH6 and ATF2 in the progression and development of HCC remain largely elusive.

There are some limitations in this study. For example, how MARCH6 regulated ATF2 was unclear. MARCH6 


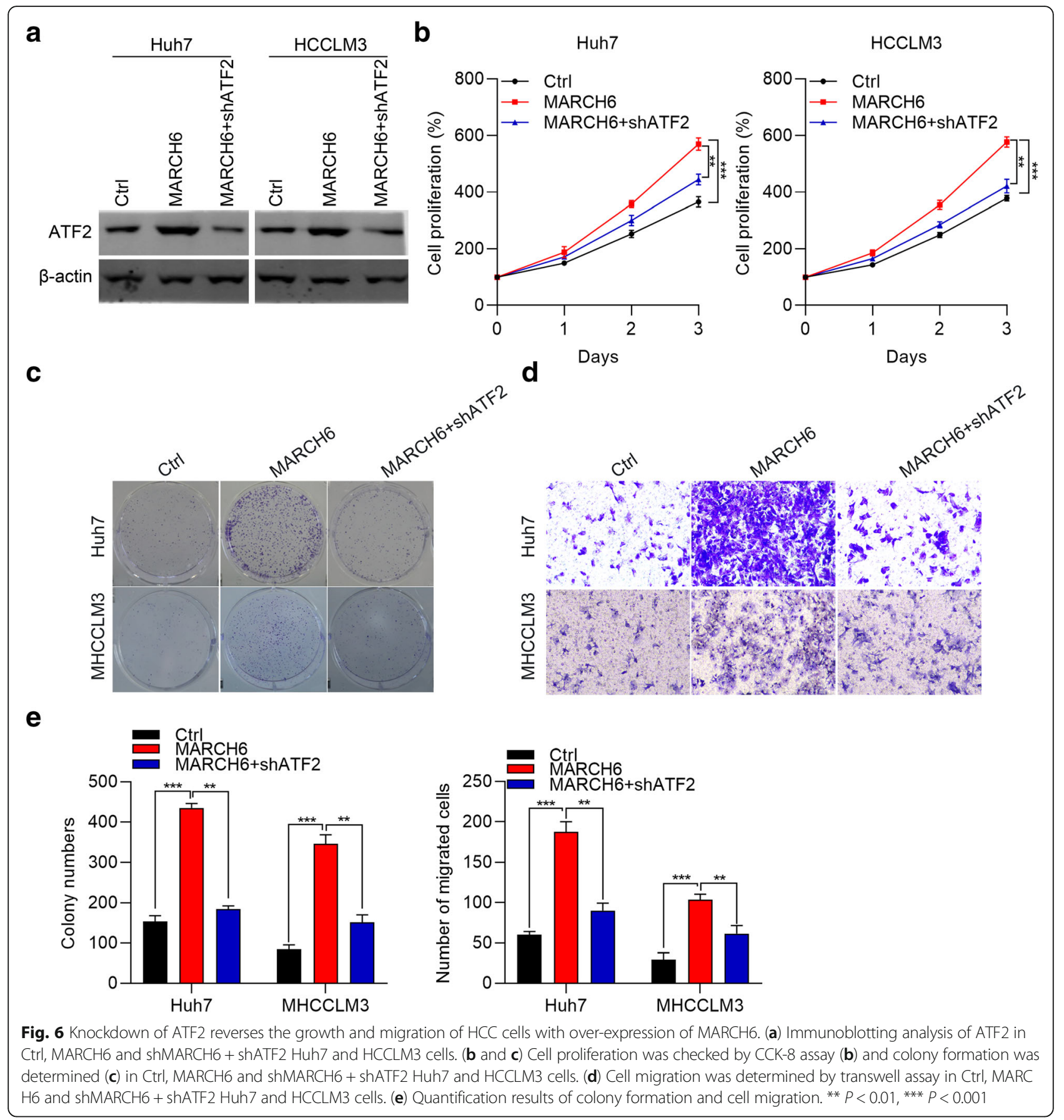

is an E3 ligase which degrades the abundance of downstream substrate via interaction. However, MARCH6 potentiates ATF2 at both mRNA and protein level, suggesting that MARCH6 may not interact with ATF2. Additionally, whether MARCH6 interacts with and regulates the upstream factors of ATF2 needs further studies.

\section{Conclusion}

In conclusion, our study showed that MARCH6 was upregulated in HCC. In addition, MARCH6 overexpression promoted the proliferative and invasive ability of $\mathrm{HCC}$ cells by potentiating the expression of ATF2, suggesting that ectopic MARCH6 expression may be an important driver for HCC development. MARCH6 may be a promising target for the diagnosis and treatment of HCC.

\section{Abbreviations}

HCC: Hepatocellular carcinoma; MARCH6: Membrane associated ring-CH-type finger 6; ATF2: Activating transcription factor 2; AR: Androgen receptor; TCGA: The Cancer Genome Atlas; SDS-PAGE: Sodium dodecyl sulfatepolyacrylamide gel electrophoresis 


\section{Supplementary Information}

The online version contains supplementary material available at https://doi. org/10.1186/s12885-021-08540-x.

Additional file 1: Supplementary Fig. 1. MARCH6 is negatively associated with pro-apoptotic proteins.

\section{Acknowledgments}

Not Applicable.

\section{Authors' contributions}

J.W., P.Z. and Y.L. designed the study. J.S., Z.D. and Z.C. performed most of the experiments and analyzed the data. H.L. constructed vectors and packaged lentivirus. Q.J., D.Z., S.L., X.J., D.W. and A.G. provide assistance for experiments and data analysis. J.S., J.W., P.Z. and Y.L. wrote and revised the manuscript. All authors have read and approved the manuscript.

\section{Funding}

This study was supported by National Natural Science Foundation of China (No. 81672467, 81672368, 81501652), Natural Science Foundation of Beijing (No.7172207, 5162026, 7202193) and Ministry of Science and Technology of China (No. 2018ZX09J18107). The funders mentioned above supported the experiment materials but not the experiment design, data analysis and manuscript writing.

\section{Availability of data and materials}

The data generated in this study are included in this published article.

\section{Declarations}

\section{Ethics approval and consent to participate}

The studies involved in HCC patients were carried out following the ethical standards of the institutional and/or national research committee and with the 1964 Helsinki declaration and its later amendments or comparable ethical standards. Written informed consent was obtained from each patient and the clinical study was approved by the Ethics Committee of The Fifth Medical Center of PLA General Hospital. Animal experiments were performed according the provisions of the Declaration of Helsinki and were approved by the Ethical Committee for Animal Experimentation of The Fifth Medical Center of PLA General Hospital.

\section{Consent for publication}

Not Applicable.

\section{Competing interests}

The authors declare no conflicts of interest.

\section{Author details}

${ }^{1}$ Comprehensive Liver Cancer Center, The Fifth Medical Center of PLA General Hospital, Beijing 100039, China. ${ }^{2}$ Department of General Surgery, The Fifth Medical Center of PLA General Hospital, Beijing 100039, China. ${ }^{3}$ Beijing Syngentech Co., Ltd., Beijing 102206, China. ${ }^{4}$ Research Center for Clinical and Translational Medicine, The Fifth Medical Center of PLA General Hospital, Beijing 100039, China. ${ }^{5}$ Department of Infectious Diseases, The Fifth Medical Center of PLA General Hospital, Beijing 100039, China. 'State key Laboratory of Toxicology and Medical Countermeasures, Beijing Institute of Pharmacology and Toxicology, Beijing 100850, China.

Received: 12 January 2021 Accepted: 28 June 2021 Published online: 17 July 2021

\section{References}

1. Thorgeirsson SS, Grisham JW. Molecular pathogenesis of human hepatocellular carcinoma. Nat Genet. 2002;31(4):339-46. https://doi.org/10.1 038/ng0802-339.

2. Farazi PA, DePinho RA. Hepatocellular carcinoma pathogenesis: from genes to environment. Nat Rev Cancer. 2006;6(9):674-87. https://doi.org/10.1038/ nrc1934.
3. Bulut $G B$, Sulahian $R$, Yao H, Huang $\sqcup$. Cbl ubiquitination of p85 is essential for Epo-induced EpoR endocytosis. Blood. 2013;122(24):3964-72. https://doi. org/10.1182/blood-2013-05-506212.

4. Colicelli J. Signal transduction: RABGEF1 fingers RAS for ubiquitination. Curr Biol. 2010;20(15):R630-2. https://doi.org/10.1016/j.cub.2010.06.019.

5. Wang $Y$, Zhu WG, Zhao Y. Autophagy regulates DNA repair by modulating histone ubiquitination. Mol Cell Oncol. 2016;3(5):e1214772. https://doi.org/1 0.1080/23723556.2016.1214772.

6. Kao SH, Wu HT, Wu KJ. Ubiquitination by HUWE1 in tumorigenesis and beyond. J Biomed Sci. 2018;25(1):67. https://doi.org/10.1186/s12929-018-04 70-0.

7. Berndsen CE, Wolberger C. New insights into ubiquitin E3 ligase mechanism. Nat Struct Mol Biol. 2014;21(4):301-7. https://doi.org/10.1038/ nsmb. 2780 .

8. Lehner PJ, Hoer S, Dodd R, Duncan LM. Downregulation of cell surface receptors by the $\mathrm{K} 3$ family of viral and cellular ubiquitin E3 ligases. Immunol Rev. 2005;207(1):112-25. https://doi.org/10.1111/j.0105-2896.2 005.00314.x.

9. Morrow JK, Lin HK, Sun SC, Zhang S. Targeting ubiquitination for cancer therapies. Future Med Chem. 2015;7(17):2333-50. https://doi.org/10.4155/ fmc.15.148.

10. van de Kooij B, Verbrugge I, de Vries E, Gijsen M, Montserrat V, Maas C, et al. Ubiquitination by the membrane-associated RING-CH-8 (MARCH-8) ligase controls steady-state cell surface expression of tumor necrosis factor-related apoptosis inducing ligand (TRAIL) receptor 1. J Biol Chem. 2013;288(9):661728. https://doi.org/10.1074/jbc.M112.448209.

11. Kreft SG, Wang L, Hochstrasser M. Membrane topology of the yeast endoplasmic reticulum-localized ubiquitin ligase Doa10 and comparison with its human ortholog TEB4 (MARCH-VI). J Biol Chem. 2006;281(8):464653. https://doi.org/10.1074/jbc.M512215200.

12. Nakamura N, Harada K, Kato M, Hirose S. Ubiquitin-specific protease 19 regulates the stability of the E3 ubiquitin ligase MARCH6. Exp Cell Res. 2014; 328(1):207-16. https://doi.org/10.1016/j.yexcr.2014.07.025

13. Coates HW, Chua NK, Brown AJ. Consulting prostate cancer cohort data uncovers transcriptional control: regulation of the MARCH6 gene. Biochim Biophys Acta Mol Cell Biol Lipids. 2019;1864(11):1656-68. https://doi.org/10.1 016/j.bbalip.2019.08.006

14. Loregger A, Cook EC, Nelson JK, Moeton M, Sharpe $\amalg$, Engberg S, et al. A MARCH6 and IDOL E3 ubiquitin ligase circuit uncouples cholesterol synthesis from lipoprotein uptake in hepatocytes. Mol Cell Biol. 2016;36(2): 285-94. https://doi.org/10.1128/MCB.00890-15.

15. Lopez-Bergami $P$, Lau E, Ronai Z. Emerging roles of ATF2 and the dynamic AP1 network in cancer. Nat Rev Cancer. 2010;10(1):65-76. https://doi.org/1 $0.1038 /$ nrc2681.

16. Huebner K, Procházka J, Monteiro A, Mahadevan V, Schneider-Stock R. The activating transcription factor 2: an influencer of cancer progression. Mutagenesis. 2019;34(5-6):375-89. https://doi.org/10.1093/mutage/gez041.

17. Watson G, Ronai Z, Lau E. ATF2, a paradigm of the multifaceted regulation of transcription factors in biology and disease. Pharmacol Res. 2017:119: 347-57. https://doi.org/10.1016/j.phrs.2017.02.004.

18. Giannoudis A, Malki M, Rudraraju B, Mohhamed H, Menon S, Liloglou T, et al. Activating transcription factor-2 (ATF2) is a key determinant of resistance to endocrine treatment in an in vitro model of breast cancer. Breast Cancer Res. 2020;22(1):126. https://doi.org/10.1186/s13058-020-01359-7.

19. Lau E, Feng Y, Claps G, Fukuda M, Perlina A, Donn D, et al. The transcription factor ATF2 promotes melanoma metastasis by suppressing protein fucosylation. Sci Signal. 2015;8(406):ra124.

20. Liu J, Li K, Wang R, Chen S, Wu J, Li X, et al. The interplay between ATF2 and NEAT1 contributes to lung adenocarcinoma progression. Cancer Cell Int. 2020;20(1):594. https://doi.org/10.1186/s12935-020-01697-8.

21. Chen C, Aihemaiti M, Zhang X, Qu H, Sun QL, He QS, et al. Downregulation of histone demethylase JMJD1C inhibits colorectal cancer metastasis through targeting ATF2. Am J Cancer Res. 2018;8(5):852-65.

22. Du G, Wang J, Zhang T, Ding Q, Jia X, Zhao X, et al. Targeting Src family kinase member Fyn by Saracatinib attenuated liver fibrosis in vitro and in vivo. Cell Death Dis. 2020;11(2):118. https://doi.org/10.1038/s41419-02 0-2229-2.

23. Yang B, Wang S, Xie H, Wang C, Gao X, Rong Y, et al. KIF18B promotes hepatocellular carcinoma progression through activating Wnt/ $\beta$-cateninsignaling pathway. J Cell Physiol. 2020;235(10):6507-14. https://doi.org/10.1 002/jcp.29444 
24. Wang C, Hu J, Chen Z, Wang Y, Lu S, Zhang Y, et al. Reversibility of hATMSCs phenotypic and metabolic changes after exposure to and withdrawal from HCC-conditioned medium through regulation of the ROS/MAPK/HIF1a signaling pathway. Stem Cell Res Ther. 2020;11(1):506. https://doi.org/1 0.1186/s13287-020-02010-0

25. Tao R, Wang C, Lu Y, Zhang C, Zhou H, Chen $\mathrm{H}$, et al. Characterization and cytotoxicity of Polyprenol lipid and vitamin E-TPGS hybrid nanoparticles for Betulinic acid and low-substituted hydroxyl Fullerenol in MHCC97H and L02 cells. Int J Nanomedicine. 2020;15:2733-49. https://doi.org/10.2147/IJN.S24 9773.

26. Alqahtani A, Khan Z, Alloghbi A, Said Ahmed TS, Ashraf M, Hammouda DM Hepatocellular Carcinoma: Molecular Mechanisms and Targeted Therapies. Medicina (Kaunas). 2019;55(9):526. https://doi.org/10.3390/medicina55090526.

27. Njei B, Rotman Y, Ditah I, Lim JK. Emerging trends in hepatocellular carcinoma incidence and mortality. Hepatology. 2015;61(1):191-9. https:// doi.org/10.1002/hep.27388

28. Miller KD, Siegel RL, Lin CC, Mariotto AB, Kramer JL, Rowland JH, et al. Cancer treatment and survivorship statistics, 2016. CA Cancer J Clin. 2016; 66(4):271-89. https://doi.org/10.3322/caac.21349.

29. El-Serag HB. Hepatocellular carcinoma: an epidemiologic view. J Clin Gastroenterol. 2002;35(5 Suppl 2):S72-8. https://doi.org/10.1097/00004836-2 00211002-00002

30. Gallo LH, Ko J, Donoghue DJ. The importance of regulatory ubiquitination in cancer and metastasis. Cell Cycle. 2017;16(7):634-48. https://doi.org/10.1 080/15384101.2017.1288326.

31. Shen N, Wang Y, Zhou W, Wang Z, Tu Q, Jiang W. Inhibition of cellular proliferation by knockdown of MARCH6 gene expression in breast cancer cells. Zhong Nan Da Xue Xue Bao Yi Xue Ban. 2016;41(10):1009-15. https:// doi.org/10.11817/j.issn.1672-7347.2016.10.001.

32. Zelcer N, Sharpe L, Loregger A, Kristiana I, Cook EC, Phan L, et al. The E3 ubiquitin ligase MARCH6 degrades squalene monooxygenase and affects 3hydroxy-3-methyl-glutaryl coenzyme a reductase and the cholesterol synthesis pathway. Mol Cell Biol. 2014;34(7):1262-70. https://doi.org/10.112 8/MCB.01140-13

\section{Publisher's Note}

Springer Nature remains neutral with regard to jurisdictional claims in published maps and institutional affiliations.

Ready to submit your research? Choose BMC and benefit from:

- fast, convenient online submission

- thorough peer review by experienced researchers in your field

- rapid publication on acceptance

- support for research data, including large and complex data types

- gold Open Access which fosters wider collaboration and increased citations

- maximum visibility for your research: over $100 \mathrm{M}$ website views per year

At $\mathrm{BMC}$, research is always in progress.

Learn more biomedcentral.com/submissions 